\title{
Inner Wall of the Vestibule
}

National Cancer Institute

\section{Source}

National Cancer Institute. Inner Wall of the Vestibule. NCI Thesaurus. Code C32813.

The wall facing the inside of the vestibule of the inner ear. 